\title{
Clinical Epidemiological Study of Oral Pre-cancer and Cancer in a Tertiary/Referral Hospital
}

\author{
Sadaksharam Jayachandran
}

Professor and Head, Department of Oral Medicine and Radiology

Tamil Nadu Government Dental College \& Hospital, Chennai.

\begin{abstract}
Aim: To study the prevalence of oral cancer in patients reported to the Department of Oral Medicine and Radiology, Tamil Nadu Government Dental College and Hospital.

Materials and Methods: After selecting the patients with the clinical criteria the history of the habits, duration in months and frequency per day, duration of the symptoms, oral mucosal site involvement, lymph node involvement, associated with lesions were recorded in a structured proforma. Incisional biopsy under Local anaesthesia and histological examination was performed. The results obtained were analysed statistically.
\end{abstract}

Results: 200 patients were diagnosed with oral cancer, $49 \%$ were male and $51 \%$ were females. In that 51(25\%) were associated with premalignant lesions, 94 had only one habit, 74 had a combination of habits, $40 \%$ were histopathologically moderately differentiated followed by $33 \%$ poorly differentiated and $27 \%$ well differentiated. And patients with stage III $(31 \%)$ and IV $(43 \%)$ were reported predominantly than stage I $(12 \%)$ and II $(14 \%)$.

Conclusion: Early diagnosis and intervention prevent the progression of oral cancer.

Keywords : Tobacco, awareness, oral cancer, epidemiology.

Correspondence : Dr. Sadaksharam Jayachandran, Professor and Head, Department of Oral Medicine and Radiology, Tamil Nadu Government Dental College \& Hospital, Chennai - 600003 , Mob. no.: +919444185662, E-mail: drsjayachandranmds@yahoo.com. 
156 Clinical Epidemiological study of Oral pre-cancer and cancer in a Tertiary/Referral Hospital

\section{INTRODUCTION}

Oral carcinoma is a global health problem with increasing prevalence and mortality rates. It is the sixth most common cancer in the world (1). Worldwide, the annual incidence exceeds $3,000,000$ new cases (2). Oral cancer accounts for $2 \%$ cancer death in males and $1 \%$ in females. Majority of oral cancers involve tongue, oropharynx and floor of the mouth. The lips, gingiva, dorsum of the tongue and palate are less common sites (3). Oral cancer is a disease of increasing age. Approximately $95 \%$ of cases occur in people older than 40 years (1). The age related incidence suggests that time dependent factors results in initiation and progression of genetic events that results in malignant change. The incidence of oral cancer is clearly age related which may reflect declining immune surveillance with age, time for accumulation of genetic changes and duration of exposure to initiators and promoters.

Tobacco and alcohol are acknowledged with factors for oral cancers. In parts of Asia, for example India where the use of tobacco, betel nut or lime to form a quid is widespread, the incidence of oral cancer is high. The use of smokeless tobacco products (chewing tobacco and snuff) is of increasing concern due to increase in their use at young age $(4,5,6)$.

Benign hyperkeratosis and epithelial dysplasia have been documented after short term use and it is likely that chronic use will be associated with an increase in incidence of malignant lesions (7).

All forms of alcohol including hard liquor, wine and beer have been implicated in etiology of oral cancer. The combined effect of alcohol and tobacco result in synergistic effect on the development of oral cancer (6). The mechanism may include dehydrating effects of alcohol on the mucosa, increasing mucosal permeability and the effect of carcinogens contained in alcohol/tobacco. In lip cancer, sun exposure and tendency to burn, pipe smoking and alcohol are identified risk factors.

Oral cancer is a result of a multistage process from normal to dysplastic lesions and ultimately to carcinoma. Dysplastic lesions have been categorized as mild, moderate or severe based on histologic criteria. Carcinoma in situ is a lesion in which abnormal cells involve the entire epithelium without invasion through the basement membrane (8). The presence and severity of dysplasia is thought to have an impact on the malignant risk of potentially malignant disorders (7).

Oral cancer is initially asymptomatic and symptoms develop after progression of disease. Discomfort is the most common symptom that leads the patient to seek care and is present at the time of diagnosis in up to $85 \%$ of the patients. Patients also may present with an awareness of a mass in the mouth or neck. 
Dysphagia, odynophagia, otalgia, limited movement and bleeding occur less frequently. Lymphatic spread of oral carcinoma usually involves the submandibular and digastrics nodes, the upper deep cervical nodes and finally the remaining nodes along the cervical chain (9).

Imaging including routine radiology, computed tomography, nuclear scintigraphy, magnetic resonance imaging and ultrasonography can provide evidence of bone involvement and can indicate the extent of some soft tissue lesions.

The principle objective of treatment is to cure cancer patients. The choice of treatment depends on such factor as cell type and degree of differentiation. Surgery/radiation are used with curative intent in the treatment of oral cancer. Chemotherapy is an adjuvant to principle therapeutic modalities of radiation and surgery.

\section{Materials and method:}

The study was conducted at the Department of Oral Medicine and Radiology, Tamil Nadu Government Dental College and Hospital, Chennai, after obtaining the ethical clearance. The study population was screened from the patients visiting the Department of Oral Medicine and Radiology TNGDC\&H. After selecting the patients with the positive history of betel quid, tobacco, alcohol use, pain, swelling, ulceration, growth, white /red patches, numbness, difficulty in swallowing and chewing, s o c i o-d e m o graphic-economic characteristics, past history as well as informed consent were obtained from each patient after explaining the complete detail about the study. Complete clinical examination (intra and extra oral examination) by using diagnostic instruments was performed. Laboratory investigations including routine blood (CBC, $\mathrm{Hb} \%$, ESR, Peripheral smear), urine analysis (urine glucose, protein and deposits) and ICTC for HIV screening was done. The history of habits, especially with reference to the duration in months and frequency per day, duration of the symptoms, oral mucosal site involvement, lymph node involvement, associated with lesions were recorded in a structured proforma specially designed for the study. The results were analysed statistically.

The parameters used in the establishment of the diagnosis are positive history of the use of betel quid, tobacco and alcohol. Besides symptoms and signs of pain, swelling, ulceration, growth on oral mucosal sites alongwith the involvement of lymph nodes and the associated potentially malignant disorders were recorded in a structured proforma designed for the study.

Radiological examinations were carried to evaluate bone involvement. Patients with diffuse and multifocal lesions were subjected to toluidine blue staining. The patients were then subjected to incisional biopsy under Local anaesthesia and histological examination 
158 Clinical Epidemiological study of Oral pre-cancer and cancer in a Tertiary/Referral Hospital

was performed.

After the histopathological result, the patients were sent to the Department of Oncology for further management. Necessary treatment was given to patients with oral precancer and cancer.

\section{Results:}

200 patients were diagnosed with oral cancer. Their ages ranged from 30-80 years $(36 \%)$. The age of 72 patients were between 30-50 years, 75 patients were between $50-60$ years $(37 \%)$ and 53 patients were more than 60 years $(27 \%)$.

Among the 200 patients, 98 patients were male (49\%) and 102 patients were female $(51 \%)$. Regarding the distribution of habits in patients included in the study, 32 patients had no habits, 75 chewed quid, 26 chewed pan parag, 45 were smokers and 22 were alcoholic. Among the patients who had the history of habits, 94 had only one habit, 74 had a combination of habits [Chewing + alcohol + smoking (23), Chewing + alcohol (19), Chewing + smoking (32)] (Table 1).

Table 1: Distribution of Risk Habits

\begin{tabular}{|l|c|c|}
\hline \multicolumn{1}{|c|}{ Habits } & No of patients(n=200) & Percentage \\
\hline No habit & 32 & $16 \%$ \\
\hline Quid & 75 & $38 \%$ \\
\hline Pan Parag & 26 & $13 \%$ \\
\hline Smoking & 45 & $22 \%$ \\
\hline Alcohol & 22 & $11 \%$ \\
\hline Only one habit & 94 & $47 \%$ \\
\hline Combination of habits & 74 & $37 \%$ \\
1.Chewing + alcohol + smoking & 23 & $11.5 \%$ \\
2.Chewing + alcohol & 19 & $9.5 \%$ \\
3.Chewing + smoking & 32 & $16 \%$ \\
\hline
\end{tabular}

Regarding the oral hygiene practise of the patients included in the study, we have found that 70 were using brush to cleanse the tooth, 91 were using fingers and 39 were using stick. The frequency of habit among them was 149 were brushing once daily, 25 twice daily and 26 don't brush regularly.

Out of 200 patients, on careful clinical examination, 51 were found to have associated with premalignant lesions (Fig-1a,1b,1c,1d) [Oral submucous fibrosis (31), leukoplakia (14), lichen planus (6)] and 149 had no premalignant lesion. 71 lesions $(36 \%)$ were present on Buccal Mucosa, 31 lesions (16\%) were on Tongue, 28 lesions (14\%) were on alveolar mucosa, 23 were on the floor of the mouth(11\%), 13 were on the Palatal Mucosa (6\%), 13 were on the Retromolar trigone (6\%), lip (6\%) and gingiva (5\%). Buccal Mucosa was clearly the predominately affected site among the 200 patients (Table 2 ). 


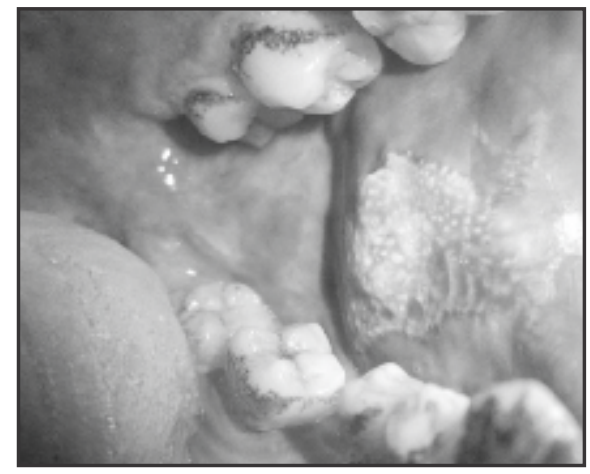

Fig. 1a: Specked leukoplakia

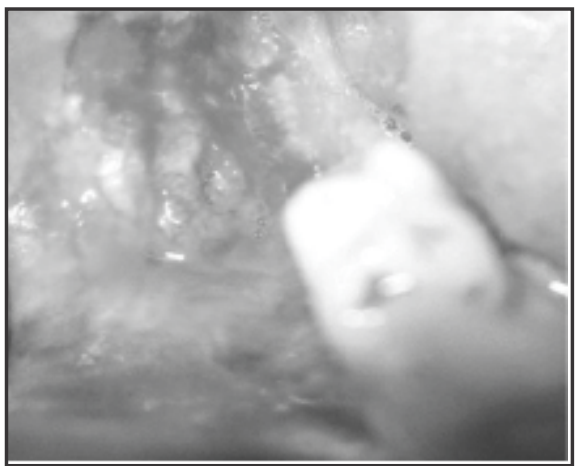

Fig. 1c: Erosive Oral lichen planus

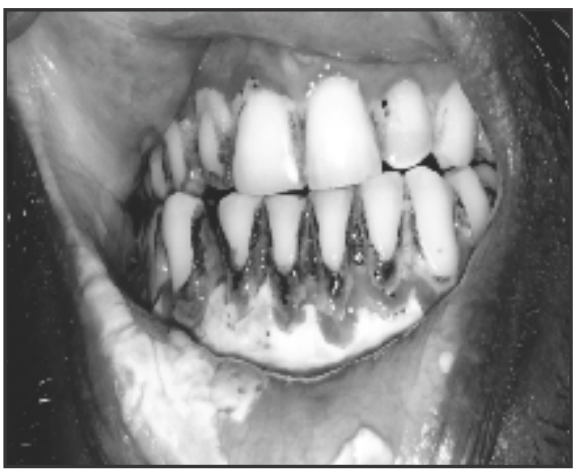

Fig. 1b: Leukoplakia

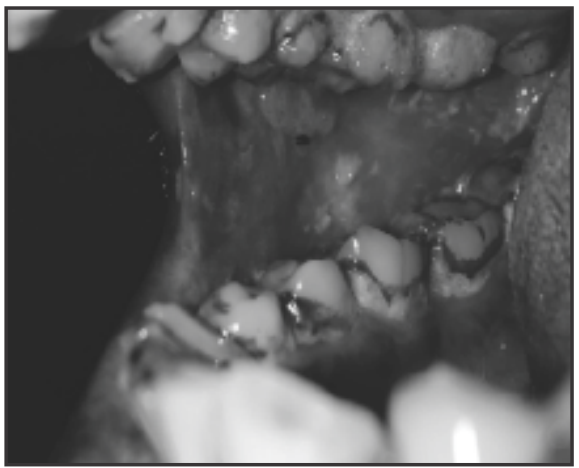

Fig. 1d: Oral submucous fibrosis

Fig. 1: Clinical pictures of potentially malignant disorders.

Table 2: Distribution of Lesions and Sites

\begin{tabular}{|c|c|c|}
\hline Associated pre malignancy & No of patients(n=200) & Percentage \\
\hline Associated & 51 & $25 \%$ \\
1. Oral submucous fibrosis & 31 & $15 \%$ \\
2. leukoplakia & 14 & $7 \%$ \\
3. lichen planus & 6 & $3 \%$ \\
Not associated & 149 & $75 \%$ \\
\hline Site & No of patients(n=200) & Percentage \\
\hline Buccal mucosa & 71 & $36 \%$ \\
Tongue & 31 & $16 \%$ \\
Alveolar Mucosa & 28 & $14 \%$ \\
Floor of the mouth & 23 & $11 \%$ \\
Palate & 13 & $6 \%$ \\
Retro molar region & 13 & $6 \%$ \\
Lip & 11 & $6 \%$ \\
Gingiva & 10 & $5 \%$ \\
\hline \multicolumn{2}{|c|}{}
\end{tabular}


160 Clinical Epidemiological study of Oral pre-cancer and cancer in a Tertiary/Referral Hospital

On through clinical examination, we have found that, 128 had involvement of submandibular lymph nodes, 32 involved the upper deep cervical and 40 had no node involvement. The submandibular lymph node showed predominant involvement than the other nodes. Regional node involvement was found to be as N0 (40), N1 (62), N2a (47), N2b (23), N2c (16), N3 (12) (Table 3).

Table 3: Lymph Node involvement and Staging of Tumor

\begin{tabular}{|c|c|c|}
\hline Lymph nodes & No of patients(n=200) & Percentage \\
\hline Sub mandibular & 128 & $64 \%$ \\
\hline Upper deep cervical & 32 & $16 \%$ \\
\hline Regional node involvement & No of patients(n=200) & Percentage \\
\hline N0 & 40 & $20 \%$ \\
\hline NI & 62 & $31 \%$ \\
\hline N2a & 47 & $23.5 \%$ \\
\hline N2b & 23 & $11.5 \%$ \\
\hline N2c & 16 & $8 \%$ \\
\hline N3 & 12 & $6 \%$ \\
\hline Staging & No of patients(n=200) & Percentage \\
\hline Stage I & 25 & $12 \%$ \\
\hline Stage II & 28 & $14 \%$ \\
\hline Stage III & 61 & $31 \%$ \\
\hline Stage IV & 86 & $43 \%$ \\
\hline
\end{tabular}

25 were diagnosed stage I, 28 as stage II, 61 as stage III and 86 as stage IV (Table 3). The patients in the stage III and IV were out numbering the patients in stage I and II. Being a tertiary care and a referral hospital, most of the reported were referred at the stage II and IV for the treatment to this hospital.

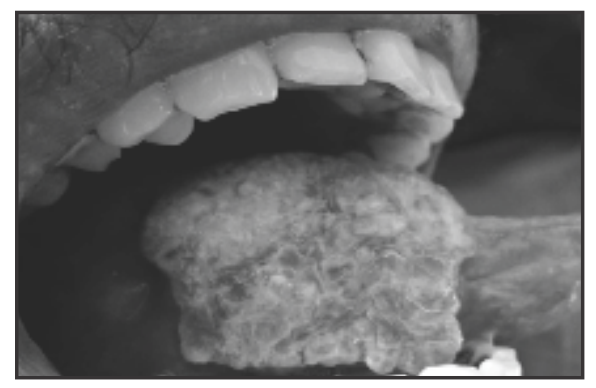

Fig. 2a: Carcinoma of tongue TNM stage III
After histopathological confirmation, among the 200 patients, 65 cases were histopathologically poorly differentiated, 55 were well differentiated (Fig-2a,2b) and 80 were moderately differentiated figure (Fig-3a, 3b) (Table 4).

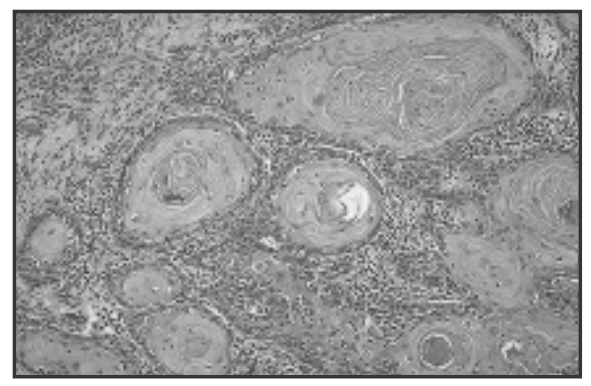

Fig. 2b: Histopathological photo

Fig. 2: Well differentiated squamous cell carcinoma. 


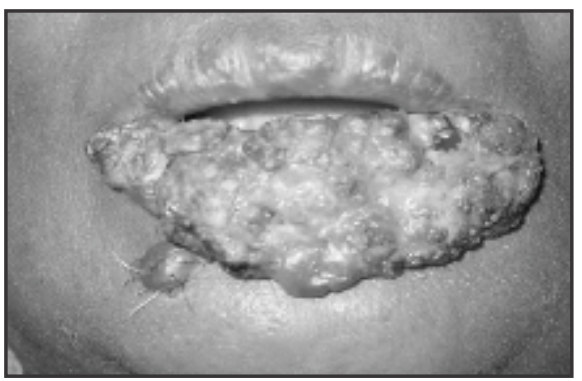

Fig. 3a: Carcinoma of lip

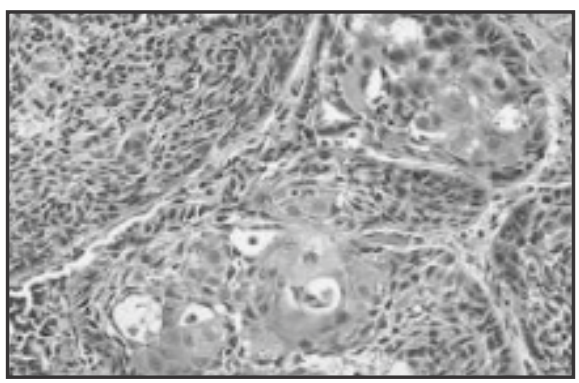

Fig. 3b: Histopathological photo

Fig. 3: Moderately differentiated squamous cell carcinoma.

Table 4: Distribution of Histopathological Grading of Squamous cell carcinoma

\begin{tabular}{|c|c|c|}
\hline Histopathology & No of patients $(\mathbf{n}=\mathbf{2 0 0})$ & Percentage \\
\hline Poorly differentiated & 65 & $33 \%$ \\
\hline Moderately differentiated & 80 & $40 \%$ \\
\hline Well differentiated & 55 & $27 \%$ \\
\hline
\end{tabular}

Among the 200 patients who were study population, $158(79 \%)$ were native of Tamil Nadu and 42(21\%) were from other states.

\section{Discussion:}

Oral carcinoma is a global health problem with increasing prevalence and mortality rates. Among these, the majority are squamous cell carcinoma. $64 \%$ of the total study population were from 50-70 years of age group and $36 \%$ of the population were from 30-50 years of age group. In the aspect of age, present study was consistent with study by Burungale $e t$ al. (10), suggesting that incidence of malignancy increases with age. But it is also found that the percentages of patients in younger age groups were also considerably affected. The age related incidence suggests that there dependent factors result in initiation and progression of genetic event that result in malignant change. The incidence of oral cancer is age related which may reflect declining immune surveillance with age.

In our data, 25 were diagnosed stage I, 28 as stage II, 61 as stage III and 86 as stage IV (Table 3 ). The patients in the stage III and IV outnumbers the patients in stage I and II. Being a tertiary care and a referral hospital, most of the reported were referred at the stage II and IV for the treatment to this hospital. Our results correlates with studies by Khandekar et al. (11) and Singh et al. (12) that the reported cancer cases were in the advanced stages i.e. stage III and IV of TNM classification and majority of them were squamous cell carcinoma. 
162 Clinical Epidemiological study of Oral pre-cancer and cancer in a Tertiary/Referral Hospital

The most important etiological factors are tobacco, excess consumption of alcohol and betel quid usage, these factors act separately or synergistically. Attributable risk of oral cancer due to both tobacco and alcohol is estimated to be more than $80 \%$ (13).

Observation in this study is similar to the study conducted Burungale et al. (10) who reported that tobacco chewing, tobacco smoking and alcohol consumption habits shows the synergistic effect in development of oral premalignant and malignant conditions.

A diet deficient in antioxidants is a further factor that predisposes towards the development of oral cancer (13).

Gabriel et al. (14) in 2006 in his study to determine the effects of smoking in the antioxidant level in serum, found that chronic smoking lowers the concentration of dietary antioxidants in serum. So from his observation he suggested that smoking as the risk factor for oral cancer.

Reactive oxygen species are highly reactive molecules that have been implicated in the etiology of potentially malignant disorders. The protective mechanism exerted by the antioxidants is by preventing the production of free radicals and repairing the damage. When the level of free radicals exceeds the antioxidant level disease will result (15).

Babu S et al. (16) in 1996 from his study revealed that habitual chewing of pan masala is associated with earlier presentation of potentially malignant conditions.

In this study, cases showed poor oral hygiene which is similar to the study performed by Anirudh Shukla (17) who reported that in $29.41 \%$ of the cases had poor oral hygiene.

In this study, among the premalignant disorders oral submucous fibrosis accounts for highest prevalence followed by leukoplakia and least being lichen planus which is similar to the studies conducted by Anirudh Shukla (17). Buccal mucosa was the commonest site among both oral premalignant and malignant conditions which is consistent with studies by Anirudh Shukla (17) and Burungale et al.(10).

Finding out the source and planning for health awareness activities for the prevention are the major role of the epidemiological studies. Epidemiological studies will be helpful in planning future health care awareness activities, tobacco cessation counselling needed towards prevention of oral cancer. This epidemiological study will be helpful to the patients as it will reduce the morbidity, reduce the incidence of invasive oral cancer, improve the prognosis of individual patients and also helps in identification of high risk group and opportunities for intervention (18).

After the diagnosis of oral cancer, the survival rate of the patients are 50$55 \%$, even less in patients when diagnosed 
at the stage III and stage IV. Prevention of oral cancer and its mortality depends upon the early detection of the lesion. For this the dental professionals have to update their knowledge regarding the oral cancer and its prevention and early diagnosis. And it is also emphasized on them, the importance of thorough multiple oral examination and obtaining the complete history from the patient (19).

Since there are enormous evidences to support the relation between the oral diseases and oral habits, controlling and preventing the tobacco usage is now gaining greater significance. In tobacco cessation counselling, in addition to asking and advising the patients regarding the ill effects of habits, steps should be taken for the intervention of the habit (i.e.) $5 \mathrm{As}$ ' protocol should be followed, asking about the smoking status, advising the benefits of quitting, assessing the motivation to quit, assisting in the quitting attempt and arranging for supportive follow-up (20).

\section{Conclusion:}

Creating awareness about the evil effect of chewing and/or smoking form of tobacco and about the early precancerous lesions and conditions, self- examination of the mouth is essential for preventing the occurrence of oral cancer.

Early diagnosis and intervention prevent the progression of oral cancer. In general, the early signs and warnings of oral cancer should be displayed in dental hospitals for patient education. Dental surgeons play a vital role in the prevention of oral cancer by educating the patients on various bad effects of tobacco chewing, snuff and alcohol use. The dental surgeons should also have a thorough knowledge about the diagnosis of various precancerous lesions and conditions, investigations and the treatment modalities for quick intervention. They should also know the referral protocol if they diagnose frank malignancy.

Tobacco cessation counselling centres should be initiated in dental hospitals for counselling the patients.

\section{REFERENCES:}

1. Warnakulasuriya S (2009). Global epidemiology of oral and oropharyngeal cancer. Oral Oncol 45(4):309-316.

2. Mehrotra R, Pandya S, Chaudhary AK, Kumar M, Singh M (2008). Prevalence of oral pre-malignant and malignant lesions at a tertiary level hospital in Allahabad, India. Asian Pac J Cancer Prev 9(2):263265.

3. Sheikh S, D'souza J (2012). A case of well-differentiated squamous cell carcinoma in an extraction socket. $J$ Indian Soc Periodontol 16:602-605.

4. La Vecchia C, Tavani A, Franceschi S, Levi F, Corrao G, Negri E (1997). Epidemiology and prevention of oral cancer. Oral Oncol 33: 302-312. 
164 Clinical Epidemiological study of Oral pre-cancer and cancer in a Tertiary/Referral Hospital

5. Blot WJ, McLaughlin JK, Winn DM (1988). Smoking and drinking in relation to oral and pharyngeal cancer. Cancer Res 48: 3282-3287.

6. Ko YC, Huang YL, Lee $\mathrm{CH}$, Chen MJ, Lin LM, Tsai CC (1995). Betel quid chewing, cigarette smoking and alcohol consumption related to oral cancer in Taiwan. J Oral Pathol Med 24(10):450-453.

7. Shirani S, Kargahi N, Razavi SM, Homayoni S (2014). Epithelial Dysplasia in Oral Cavity. IJMS 39(5):406-417.

8. Glick M, Feagans WM. Burkets Oral Medicine. 12th edition. USA Shelton. Peoples Medical Publishing House, 2015.

9. Neville BW, Day TA (2002). Oral cancer and precancerous lesions. $C A$ Cancer J Clin 52(4): 195-215.

10. Burungale SU, Durge PM, Burungale DS, Zambare MB (2014). Epidemiological Study of Premalignant and Malignant Lesions of the Oral Cavity. JAIR 2(9):519-523.

11. Khandekar SP, Bagdey PS, Tiwari RR (2006). Oral Cancer and Some Epidemiological Factors : A Hospital Based Study. Indian Journal of Community Medicine 31(3):157-159.
12. Singh MP, Misra S, Rathanaswamy SP, et al. (2015). Clinical profile and epidemiological factors of oral cancer patients from North India. Natl J Maxillofac Surg 6:21-24.

13. Petridou E, Zavras AI, Lefatzis D (2002). The role of diet and specific micronutrients in the etiology of oral carcinoma. Cancer 94: 2981-2988.

14. Gabriel HE, Liu Z, Crott JW, et al. (2006). A comparison of carotenoids, retinoids, and tocopherols in the serum and buccal mucosa of chronic cigarette smokers versus nonsmokers. Cancer Epidemiol Biomarkers Prev 15(5):993-999.

15. Rodrigo R. Oxidative stress and antioxidants: their role in human disease. Nova Science Publishers: New York; 2009.

16. Babu S, Bhat RV, Kumar PU, et al. (1996). A comparative clinicopathological study of oral submucous fibrosis in habitual chewers of pan masala and betel quid. J Toxicol Clin Toxicol 34(3):317-322.

17. Shukla A (2014). Potentially Malignant Disorders of the Oral Cavity: A Clinical Study. Indian J Otolaryngol Head Neck Surg 66(1):79-85. 
18. Zain RB, Ghazali N (2001). A review of epidemiological studies of oral cancer and precancer in Malaysia. Annal Dent Univ Malaya 8: $50-56$.

19. Messadi DV, Wilder-Smith P, Wolinsky L (2009). Improving Oral Cancer Survival: The Role of Dental
Providers. J Calif Dent Assoc 37(11):789-798.

20. Chestnutt IG, Binnie V (1995). Smoking cessation counselling--a role for the dental profession? $\mathrm{Br}$ DentJ 179(11):411-415. 